\title{
COMENTARIO EDITORIAL: Definición de prioridades para la implementación de intervenciones sanitarias: enfermedades transmisibles (última entrega)
}

Priority setting for the implementation of sanitary interventions: communicable diseases (final article)

Adolfo Rubinstein*

\begin{abstract}
Resumen
Este artículo describe el impacto sanitario en Argentina y algunos países de lationamérica, de tres enfermedades transmisibles: tuberculosis, infección por el virus de la inmunodeficiencia humana $(\mathrm{VIH})$ y enfermedad de Chagas o tripanosomiasis americana.

Utilizando como ejemplo los resultados de una evaluación de la costo-efectividad de distintas intervenciones para reducir la carga de enfermedad cardiovascular en la Ciudad de Buenos Aires, el autor también reflexiona sobre las mejores formas de asignar recursos para las diferentes intervenciones sanitarias y explica sucintamente la taxonomía de los diferentes tipos de evaluaciones económicas en salud.

\section{Abstract}

This article describes the health impact of three communicable diseases in Argentina and some latin-american countries: tuberculosis, HIV infection and Chagas disease or american trypanosomiasis. Using a cost-effectiveness evaluation of different interventions to reduce the burden of cardiovascular disease in the city of Buenos Aires as an example, the author also mentions some of the best resource allocation strategies for different health interventions and explains the taxonomy of economic evaluations.
\end{abstract}

Palabras clave: tuberculosis, virus de la inmunodeficiencia humana, enfermedad de Chagas, tripanosomiasis americana, costo-efectividad. Key words: tuberculosis, human inmunodeficiency virus, Chagas disease, american trypanosomiasis

Rubisntein A. Definición de prioridades para la implementación de intervenciones sanitarias: enfermedades transmisibles (última entrega). Evid. actual. práct. ambul; 10(6):162-165, Nov-dic.2007.

\section{Tuberculosis}

La tuberculosis es una de las enfermedades infecciosas que más muertes provoca en el mundo y en Argentina. En los últimos 15 años su incidencia se incrementó, principalmente por su asociación con el virus de la inmunodeficiencia humana (VIH). Se estima que más del $20 \%$ de la población mundial está infectada por el M. tuberculosis. Según la Organización Mundial de la Salud (OMS) cada año ocurren en el mundo entre ocho y diez millones de casos nuevos de tuberculosis, con aproximadamente dos millones de muertes atribuidas a esta enfermedad en 2000.

Existen claros factores socioeconómicos que determinan que casi el $95 \%$ de los casos ocurra en países subdesarrollados, presentándose cerca de $80 \%$ en la población económicamente activa (15 a 59 años).

Si bien los distintos programas llevados a cabo para disminuir su impacto han mostrado un progreso indudable, especialmente en algunas regiones del mundo, mejorar sustancialmente su control dependerá de un enorme esfuerzo coordinado para lograr la expansión de los programas cortos de tratamiento supervisado (en inglés: DOTS) la prevención de nuevos casos de tuberculosis a través del manejo adecuado de los contactos, la prevención de la tuberculosis multiresistente garantizando especialmente la finalización de los tratamientos y el desarrollo de estrategias específicas de prevención y control de la tuberculosis asociada al VIH en regiones de alta prevalencia.

Debido a que es una enfermedad de reporte obligatorio y a que la entrega de los fármacos antituberculosos está supeditada a la comunicación de los casos nuevos a las autoridades sanitarias, los datos sobre la incidencia (casos nuevos/población susceptible) son conocidos.

Sin embargo, las diferencias halladas entre las distintas provincias argentinas en el reporte de 2004 hace pensar que la situación sanitaria es muy desigual o que el reporte de casos no funciona igual en todas ellas, ya que la tasa reportada en Jujuy o Salta fue siete veces mayor que la de Catamarca y, que a su vez, ésta y la de Santiago del Estero fueron similares a la de algunos países desarrollados.
Ver figuras 1 y 2

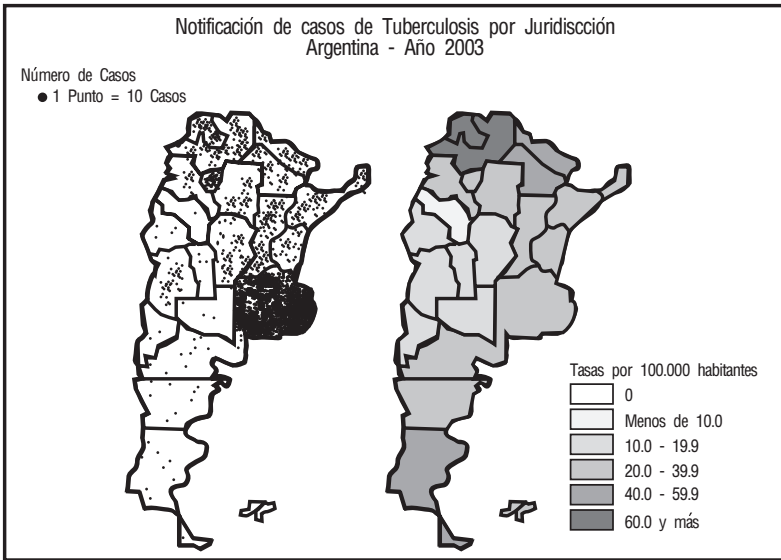

Notificación de tuberculosis de todas las formas por provincias Argentina, 2003. Tasas por 100.000 habitantes

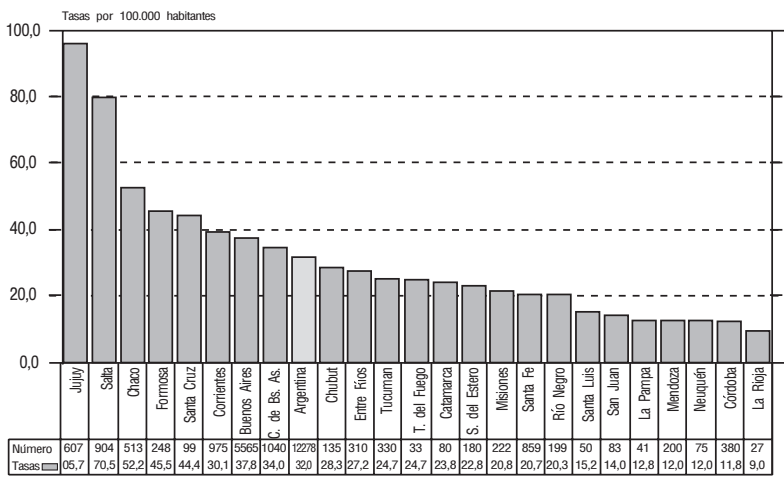

Entre 1980 y 1998 la tendencia de la morbilidad por tuberculosis en Argentina pasó por distintos estadios. La denominada variación anual promedio (VAP) de la incidencia de nuevos casos no fue significativa hasta 1983, registrándose alrededor de 17.000 casos anuales, con una ligera tendencia descendente (-0,2\% anual). Entre 1983 y 1987 el descenso fue muy importante, alcanzando una VAP de $-8,4 \%$, incluso superior a 
la de países desarrollados como EE.UU., cuya VAP para el mismo lapso fue de $-5 \%$. A partir de 1987, la tasa se estabilizó para comenzar a ascender en 1991, probablemente debido a su asociación con el VIH. Afortunadamente, esta tendencia parece controlada desde 1993.

La tasa de Argentina es seis veces superior a la de América del Norte y similar a la tasa promedio de los países del Cono Sur. La situación de Argentina es peor que la de Cuba, Chile y Uruguay, pero mejor que la de Perú, Bolivia, Brasil y Paraguay. En nuestro país, sigue ocasionando alrededor de 12.000 nuevos casos y 850 muertes anuales, representando una tasa de notificación de 31 casos y 2,2 muertes por cada 100.000 habitantes en 2004. Cuarenta y siete por ciento del total de casos nuevos corresponden a enfermos de tuberculosis pulmonar con baciloscopía positiva, representando más de 5.500 nuevos enfermos cada año y constituyendo una fuente de infección para el resto de la población.

En el subgrupo de adultos entre 15 y 29 años el incremento del riesgo de desarrollar tuberculosis fue de $36 \%$. El $80 \%$ de los casos "en exceso" fue reportado en la provincia de Buenos Aires y Capital Federal y se cree que se relaciona con la asociación entre tuberculosis y VIH. La mortalidad atribuible a tuberculosis acompañó el mismo aumento que se observó en la incidencia'. Aunque la prevalencia de tuberculosis en la población pediátrica es menos conocida que en los adultos, su patrón de infección depende del de los adultos, ya que éstos y los adolescentes actúan como reservorio. Ver figura 2.

\section{Enfermedad por el virus de la inmunodeficiencia humana}

La infección por el virus de la inmunodeficiencia humana (VIH) constituye sin dudas uno de los principales problemas de salud pública en la actualidad. Alrededor de 42 millones de personas se encuentran hoy infectadas por el virus en el mundo $y$, si bien en los últimos años la aparición de nuevos tratamientos ha permitido cambiar la perspectiva de la enfermedad y su pronóstico en el largo plazo, la inaccesibilidad de estas innovaciones para la gran mayoría de los enfermos del mundo ha contribuido a situar al VIH como la segunda causa de mortalidad de origen infeccioso, luego de las infecciones respiratorias. Esta tendencia se mantiene aun en la era en que el tratamiento antirretroviral de alta eficacia ha demostrado dramáticas reducciones en la morbi-mortalidad vinculada al VIH, lo cual es aún más impactante y doloroso.

Otro aspecto epidemiológico significativo es que hoy se reconoce que la amplia disponibilidad del tratamiento antirretroviral ha sido un condicionante fundamental de la retracción de la epidemia en el mundo desarrollado, vinculando inextricablemente al tratamiento con la prevención.

Brasil, Colombia y Argentina son los países sudamericanos que presentan la mayor cantidad de casos en la región, debido en parte a la mayor población que tienen estos países. Sin embargo, la mayor prevalencia se encuentra en los países más pequeños como Belice, Guatemala y Honduras, donde cerca del $1 \%$ de los adultos estaban infectados por el $\mathrm{VIH}$ a finales de 2003. Los factores predisponentes de la epidemia son combinaciones variables de relaciones sexuales homosexuales y heterosexuales sin protección y consumo de drogas intravenosas. En casi todos los países latinoamericanos, los niveles más altos de infección por VIH se encuentran en los hombres homosexuales ( 2 a $28 \%$ en distintas zonas) seguidos por las mujeres trabajadoras sexuales (hasta 6,3\%). Se estima que corresponden a las relaciones sexuales entre hombres el 25 al $35 \%$ de los casos de SIDA notificados en países como Argentina, Bolivia, Brasil, Guatemala y Perú.
En Argentina, la mayoría de las nuevas infecciones se ha producido como consecuencia de relaciones heterosexuales sin protección, con un creciente número de mujeres infectadas por $\mathrm{VIH}$. La razón varón-mujer entre los casos de SIDA notificados se redujo de 15:1 en 1988 a 3:1 en 2004. Sin embargo, el consumo de drogas intravenosas y las relaciones homosexuales siguen impulsando la propagación del VIH en la Argentina, especialmente en las zonas urbanas de las provincias de Buenos Aires, Córdoba y Santa Fe, donde se han producido aproximadamente $80 \%$ de los casos de SIDA. Como resultado de las pruebas efectuadas en la ciudad de Buenos Aires se encontró que un $44 \%$ de los consumidores de drogas intravenosas era positivo, mientras que en otros estudios se halló una prevalencia de $\mathrm{VIH}$ de 7 a $15 \%$ entre los hombres homosexuales ${ }^{2}$.

Se estima que en Argentina hay en la actualidad más de 120.000 personas infectadas por el $\mathrm{VIH}$, de las cuales alrededor de 27.000 han sido efectivamente diagnosticadas y notificadas al Programa Nacional de SIDA. Este hecho contribuye a sustentar la trágica paradoja de que la epidemia continúa avanzando en nuestro país -al igual que en el resto de los países en vías de desarrollo- a pesar de ser un lugar en donde el acceso a la medicación antirretroviral está garantizado por ley. Seguramente, otros factores como la insuficiencia de las estrategias de prevención y la dificultad en la implementación de los programas de detección precoz de la infección también contribuyen a esta realidad ${ }^{3}$.

\section{Enfermedad de Chagas}

La enfermedad de Chagas es una enfermedad producida por el Tripanosoma Cruzi (parásito protozoario que infecta al ser humano y a los animales domésticos y salvajes) ${ }^{4}$. Esta infección se extiende desde el sur de México hasta el norte de Argentina en forma de manchas geográficas, que a veces son bien delimitadas. Se estima un total de 16 a 18 millones de personas infectadas en 18 países de Latinoamérica. De estos, 900.000 podrían evolucionar hacia megaformaciones digestivas: megaesófago y/o megacolon y más de cinco millones hacia diversas formas de cardiopatía chagásica. Se estima una incidencia anual de 200 mil nuevos casos por año para el año 2.000 .

Se pueden evidenciar importantes diferencias entre los distintos países latinoamericanos. En Bolivia, aproximadamente $20 \%$ de la población está infectada, esto es cerca de 1,2 millones de personas; en Brasil, el porcentaje de la población infectada es de $1,3 \%$ de la población total del país, lo que significa un poco más de 2 millones de personas.

Argentina, Honduras, Paraguay y El Salvador presentan un porcentaje de 5 a $10 \%$ de la población infectada con la enfermedad, mientras que el porcentaje en Chile, Colombia, Ecuador y Venezuela está entre el 1 y el $5 \%$. Otros países como México y Nicaragua presentan un porcentaje de infección menor al $1 \%$.

En Argentina, el área de mayor endemicidad está representada por las provincias del Noroeste y las del Centro, con una superficie de $1.045 .087 \mathrm{~km} 2$ y comprendiendo a 13 estados provinciales de los 19 endémicos (los anteriores más las provincias del Noreste Argentino y Cuyo).

La prevalencia estimada de infección crónica en Argentina es de $7,5 \%(1,7 \%$ de seropositividad en los bancos de sangre de Capital Federal y $8,7 \%$ en el interior del país, con picos de hasta $42 \%$ en la provincia de Santiago del Estero). La prevalencia de esta infección ha disminuido en mujeres embarazadas, en bancos de sangre, en niños de 10 a 14 años y en jóvenes de 18 a 20 años. Para 2005 la prevalencia informada en 
menores de 15 años fue de 1,3\%. Respecto al Chagas congénito, se estiman entre 800 y 1.300 nuevos casos por año.

En función del mejor control de la transmisión vectorial, producto de las campañas de control emprendidas en Argentina en los últimos 40 años, se ha logrado disminuir la tasa global de infestación a menos de $1 \%$ en 2004 . Este conjunto de acciones se reflejó en la disminución de la prevalencia de infección en los jóvenes desde $10 \%$ en 1962 a menos de $2 \%$ en la última década. Ver figura 3.

Figura 3:

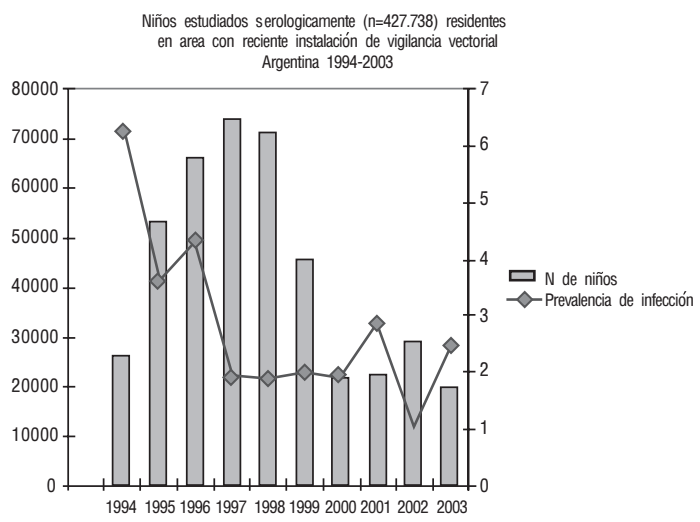

Por lo tanto, la vía de transmisión vertical de la embarazada infectada al niño como fuente de producción de nuevos casos está adquiriendo cada vez más importancia. El riesgo de transmisión perinatal (desde una madre con Chagas crónico) oscila entre el 0,5 y el $5 \%$. La prevalencia de infección en las mujeres embarazadas es de 2 al $51 \%$ en áreas urbanas de América Latina y de 23 a $81 \%$ en áreas rurales. Por lo tanto, la detección del Chagas neonatal es de capital importancia epidemiológica ya que el tratamiento oportuno con antiparasitarios en esta etapa, a diferencia del tratamiento del Chagas crónico en los adultos, es altamente efectivo en cuanto a la eliminación del parásito.

Las estrategias basadas en el control del vector con estrategias participativas, la incorporación de la detección del parásito a los controles de rutina de la mujer embarazada y el recién nacido, el control de los bancos de sangre, el diagnostico precoz y el tratamiento etiológico de la población infectada y la implementación de investigaciones operativas que ofrezcan estrategias para una vigilancia sostenida, son los pilares para reducir el impacto de esta infección como causa de muerte y discapacidad en Argentina.

Definición de prioridades para la toma de decisiones sobre la implementación de intervenciones en salud

El proceso de asignación de recursos en salud es habitualmente empírico, guiado por consideraciones políticas, sociales o financieras. La utilización de evidencia científica y económica sobre el impacto de las intervenciones, con el objeto de establecer y ordenar las prioridades para una toma de decisiones informada, que sirva para definir las políticas de cobertura, son poco comunes en los países en desarrollo.

De acuerdo a la definición original de Drummond ${ }^{5}$, las evaluaciones económicas (EE) son análisis comparativos de cursos alternativos de acción que involucran diferentes intervenciones en relación a los costos y a las consecuencias y efectos sobre la salud. Las EE pueden ser completas, si consideran tanto los costos como los efectos; o parciales si sólo consideran los costos. Los tipos de EE completa pueden categorizarse de varias maneras, pero la más utilizada las divide en cuatro tipos básicos: 1) análisis de costo-efectividad (ACE) si los efectos de las intervenciones son medidos en "unidades naturales" de ganancia de salud (años de vida ajustados por calidad o discapacidad, casos evitados, etc.); 2) análisis de costo-utilidad (ACU) Si incorpora las preferencias individuales o sociales; 3 ) análisis de costo-beneficio (ACB) si los costos y efectos son valuados en términos monetarios; 4) análisis de minimización de costos si se asume que los efectos son similares, por lo que sólo se deben comparar los costos ${ }^{5}$.

Un modelo de evaluación económica que permite analizar mejor la eficiencia con que los recursos son asignados a las diferentes intervenciones de acuerdo al beneficio agregado en salud (costo en pesos por cada año de vida ajustado por discapacidad: \$(AVAD) es el análisis de costo-efectividad generalizada o sectorial. Este método utiliza información relacionada con los niveles de costo efectividad de las intervenciones para identificar el mejor conjunto de acciones orientadas a disminuir la carga de enfermedad atribuible a diferentes condiciones.

Esta iniciativa de la Organización Mundial de la Salud, conocida como WHO-CHOICE (CHOosing Interventions that are Cost-Effective) comenzó en 1998 a recoger y analizar datos regionales de costos, incorporando los datos de carga de enfermedad, la evidencia de la efectividad de un conjunto básico de intervenciones "clave" en salud (individuales y poblacionales) en diferentes áreas: alcoholismo, salud mental, enfermedades de la infancia, enfermedades cardiovasculares, diabetes, epilepsia, VIH, contaminación ambiental, deficiencia de hierro, paludismo, salud materna y neonatal, tuberculosis, adicciones y saneamiento ambiental.

Recientemente, se llevó a cabo una evaluación económica sectorial financiada por VIGI+A, Ministerio de Salud, cuyo objetivo fue identificar el mejor conjunto de intervenciones de promoción y prevención primaria orientadas a la disminución de la carga de enfermedad cardiovascular para la población cubierta por el sistema público en la ciudad de Buenos Aires. Este estudio de costo efectividad sectorial comparó la utilidad y el costo de distintas intervenciones para mejorar la eficiencia en la asignación de los recursos para reducir la carga de enfermedad atribuible a enfermedad cardiovascular, tomando como población blanco, la población sin seguro de la ciudad de Buenos Aires ${ }^{7}$. El ranking de intervenciones a ser cubiertas de acuerdo los cocientes de costo-efectividad pueden observarse en la tabla 1.

Tabla 1: ordenamiento de intervenciones integradas para reducir la morbi-mortalidad cardiovascular en la Ciudad de Buenos Aires según su cociente Costo/Efectividad.

\begin{tabular}{l|c}
\multicolumn{1}{c|}{ Intervención } & $\begin{array}{c}\text { Costo en Pesos por } \\
\text { cada AVAD evitado } \\
\text { Menor contenido'de sal en el pan }\end{array}$ \\
\hline $\begin{array}{l}\text { Tratamiento a pacientes con riesgo cardiovascular alto (m s de } \\
\text { 20\% de riesgo de enfermedad coronaria a diez años). }\end{array}$ & $\$ 2.402$ \\
\hline $\begin{array}{l}\text { Tratamiento a pacientes de riesgo cardiovascular mayor a 10\% de } \\
\text { desarrollar enfermedad coronaria a diez años. }\end{array}$ & $\$ 3.141$ \\
\hline Campaña publicidad masiva para adopción de hábitos saludables & $\$ 6.620$ \\
\hline Terapia antihipertensiva & $\$ 7.716$ \\
\hline Tratamiento para la cesación tabáquica & $\$ 33.569$ \\
\hline Tratamiento hipolipemiante & $\$ 70.943$ \\
\hline
\end{tabular}


Fuente: Rubinstein A, García Martí S. y col. Evaluación Económica Sectorial de un paquete de intervenciones preventivas para reducir la morbimortalidad cardiovascular en la ciudad de Buenos Aires. Instituto de Efectividad Clínica y Sanitaria (IECS) y Programa VIGI+A. Ministerio de Salud de la Nación. 2005

AVAD: Año de Vida Ajustado por Discapacidad.

\section{Conclusiones}

A medida que las intervenciones y los programas para mejorar la salud en el mundo se extienden a mayores poblaciones, se vuelve cada vez más necesario contar con evaluaciones periódicas más precisas y confiables que monitoreen la salud poblacional y sus progresos.

El estudio de Carga de Enfermedad Global de 1990 puso luz sobre la importancia relativa de las enfermedades crónicas y sobre el fenómeno de la doble carga de enfermedad en los países en desarrollo, así como sobre la importancia de medir la carga atribuible a condiciones que no producen habitualmente resultados fatales, como la discapacidad atribuible a las enfermedades mentales y a injurias. El estudio de Carga de Enfermedad Global de 2001 reforzó estas conclusiones. Si se ana-lizan globalmente las tendencias en la década pasada, muchos de los avances en la reducción de la mortalidad infantil en regiones pobres se vieron contrarrestadas por el aumento de la incidencia y la mortalidad por SIDA, especialmente en los países del África Sub-sahariana. Por otro lado, el preocupante aumento de la mortalidad en adultos en Rusia y muchos otros países de Europa Central, atribuible al tabaquismo y a la epidemia de enfermedades cardiovasculares, es un recordatorio cons-tante de cómo las mejorías ganadas pueden rápidamente revertirse cuando no se implementan políticas de salud apropiadas que den cuenta de los problemas emergentes.

Muchas intervenciones eficaces, efectivas y costo-efectivas aún no se implementan a escala global a pesar de que son razonablemente financiables. Esto se debe en gran medida a la ausencia o falta de desarro-llo de sistemas de servicios de salud y de políticas públicas que faciliten su difusión y su llegada a los puntos de servicio. Para que los programas de prevención y tratamiento funcionen, los políticos y decisores sanitarios deben tener acceso a la mejor evidencia científica y económica disponible para tomar decisiones informadas que le den más "valor por dinero a los recursos invertidos, esto es salvar la mayor cantidad de vidas con la mejor calidad posible".

Lamentablemente, no disponemos aún en Argentina de datos confiables referidos a morbilidad y discapacidad atribuibles a enfermedades o factores de riesgo aunque ya se reconoce la importancia estratégica que estos merecen. De hecho, iniciativas llevadas a cabo por el Ministerio de Salud de la Nación, como la encuesta Nacional de Factores de Riesgo y la Encuesta Nacional de Nutrición, entre otras, nos comienzan a proporcionar datos que permitirán a los gobiernos elaborar políticas públicas de priorización que aseguren mejores indicadores sanitarios con mayor eficiencia y equidad.

En definitiva, de lo que se trata no es sólo de generar "datos" sino de convertir los "datos" en "información" y la información en "inteligencia" para poder tomar las decisiones más acertadas.

Recibido el 02/03/07 y aceptado el 18/11/07.

Ruiz E. Edad a la cirugía de testículo no descendido y riesgo de de cáncer testicular. Evid. actual. práct. ambul; 10(6):162-165, Nov-Dic.2007. Comentado de: Pettersson A, et al. Age at surgery for undescended testis and risk of testicular cancer. N Engl J 2007;356 (18):1835-41. PMID: 17476009 .

\section{Referencia}

1. Rubinstein F y Espinosa G. Tuberculosis en el adulto. Capítulo 118. En: Adolfo Rubinstein y Sergio Terrasa. Medicina Familiar y Práctica Ambulatoria. 2da ed. Ed.Médica Panamericana. Buenos Aires 2006

2. Hoja informativa de ONUSIDA, Nov. 2005, www.unaids.org

3. Belloso W. Virus de la inmunodeficiencia humana y SIDA. Capítulo 275. En: En: Adolfo Rubinstein y Sergio Terrasa. Medicina Familiar y Práctica Ambulatoria. 2da ed. Ed.Médica Panamericana. Buenos Aires 2006

4. Chiarpenello J, Bessone M. Capítulo 253. En: Adolfo Rubinstein y Sergio Terrasa. Medicina Familiar y Práctica Ambulatoria. 2da ed. Ed.Médica Panamericana. Buenos Aires

2006

5. Drummond y col. Methods for the Economic Evaluation of Health Care Programs. Oxford Medical Publications. 2005

6. D. Evans y col. Methods to assess the costs and health effects of interventions for improving health in developing countries. BMJ 2005;331:1137-1140

7. Rubinstein A, García Martí S. y col. Evaluación Económica Sectorial de un paquete de intervenciones preventivas para reducir la morbimortalidad cardiovascular en la ciudad de Buenos Aires. Instituto de Efectividad Clínica y Sanitaria (IECS) y Programa VIGI+A. Ministerio de Salud de la Nación. 2005

\section{INFO - EVIDENCIA}

\section{¿Qué es Evidencia, Actualización en la Práctica Ambulatoria?}

Una publicación independiente editada cada dos meses desde 1997 por la Fundación MF, organización sin fines de lucro dedicada a promover el desarrollo de la medicina familiar y la atención primaria de la salud.

\section{¿Cuál es el objetivo de Evidencia?}

Contribuir a la educación continua y a la actualización de los profesionales de la salud de la región en el área de la atención ambulatoria.

\section{Contenidos de Evidencia}

- Información independiente, actualizada y resumida en forma sencilla y en castellano sobre temas relevantes de la atención ambulatoria.

- Artículos seleccionados por su calidad y relevancia clínica, resumidos y comentados críticamente por destacados profesionales del área.

- Revisiones sobre temas clínicos, epidemiológicos, sanitarios o humanísticos, de importancia para la práctica ambulatoria.

- Notas farmacológicas para la actualización rápida de temas de terapéutica clínica.

- Discusión de casos clínicos con herramientas de Medicina Basada en la Evidencia

- Glosario de términos de epidemiología y medicina basada en la evidencia

¿Cómo es posible acceder a Evidencia?

Por suscripción a la edición en papel, o bien por Internet, en nuestro sitio: http://www.evidencia.org 